\title{
Homogeneous Discrete Time Alternating Compound Renewal Process: A Disability Insurance Application
}

\author{
Guglielmo D'Amico, ${ }^{1}$ Fulvio Gismondi, ${ }^{2}$ Jacques Janssen, ${ }^{3}$ and Raimondo Manca ${ }^{4}$ \\ ${ }^{1}$ Dipartimento di Farmacia, Università “G. d’Annunzio” di Chieti, Via dei Vestini 31, 66013 Chieti, Italy \\ ${ }^{2}$ Guglielmo Marconi University, Via Plinio 44, 00193 Roma, Italy \\ ${ }^{3}$ Solvay Business School, Universitè Libre de Bruxelles, Avenue Franklin D. Roosevelt, CP 145/1-21, 1050 Brussels, Belgium \\ ${ }^{4}$ Dipartimento di Metodi e Modelli per l'Economia, il Territorio e la Finanza, Università di Roma "La Sapienza", \\ Via del Castro Laurenziano 9, 00161 Roma, Italy \\ Correspondence should be addressed to Raimondo Manca; raimondo.manca@uniroma1.it
}

Received 19 March 2015; Revised 1 June 2015; Accepted 11 June 2015

Academic Editor: Babak Shotorban

Copyright (C) 2015 Guglielmo D’Amico et al. This is an open access article distributed under the Creative Commons Attribution License, which permits unrestricted use, distribution, and reproduction in any medium, provided the original work is properly cited.

\begin{abstract}
Discrete time alternating renewal process is a very simple tool that permits solving many real life problems. This paper, after the presentation of this tool, introduces the compound environment in the alternating process giving a systematization to this important tool. The claim costs for a temporary disability insurance contract are presented. The algorithm and an example of application are also provided.
\end{abstract}

\section{Introduction}

It is possible to assert that the roots of renewal processes are in actuarial science. Indeed, the seminal paper Lundberg [1] introduced this theory and was written by an actuary. Other two seminal papers $[2,3]$ were written by a really important probability researcher that did many applications in insurance.

For a complete introduction to the renewal processes, we refer to the book Cox [4].

As well known, renewal processes work in this way. We have a phenomenon that will be verified but we do not know when. When the studied phenomenon happens, then the system is renewed and, in homogeneous case, it restarts with the same initial characteristics (for first results in nonhomogeneous renewal processes, see Gismondi et al. [5]). It is clear that a simple actuarial model can be well simulated by this kind of stochastic process.

Suppose that we have to study a system that can assume two different values that cannot be verified together and that when one of them is not verified then the other is verified and vice versa. In this case, the consideration of only the renewal is not sufficient for the study of the problem. The random evolution of these processes can be studied by means of the alternating renewal processes that are a generalization of the renewal process (see Figure 6). These processes were applied in many fields (see, e.g., Zacks [6], Di Crescenzo et al. [7], and Elsayed [8]).

The renewal processes had and have a great relevance in Actuarial Sciences, but there were few applications of the alternating renewal processes.

In health insurance models, there are two kinds of randomness: one is given by the state of the insured (healthy or ill) and the other by the duration inside the states. This phenomenon can be well simulated by the alternating renewal processes. Both the transitions between the two states are possible. An ordinary renewal process cannot model this insurance contract. Ramsay [9] and more recently Adekambi and Mamane [10] proposed the alternating renewal process for the study of health insurance problems. The used tool 
fits well in the problem. However, the continuous time environment used in both the papers is really difficult to apply. Indeed, renewal processes in most cases should be solved numerically. For example, Adekambi and Mamane [10] constructed an interesting model in which they derive the first two moments of the aggregate claim amount of benefit paid out up to a given time $t$. The problem is that although the mathematical apparatus is adequate, the application was done in a very simple case with negative exponential probability distribution functions. D'Amico et al. [11] showed how the application of discrete time alternating renewal processes is simple.

For a general reference on the discrete time renewal processes, we recall Feller [12].

This paper will generalize the results presented in D'Amico et al. [11] applying the alternating compound renewal process for the study of insurance contracts in a discrete time environment.

Discrete time alternating compound renewal processes were described in Tijms [13] and in Beichelt [14]. In both of these books, the alternating compound processes were introduced as exercises. In Beichelt, the exercise was only described but not solved and no hints were given for solving it. In the Tijms book, an asymptotic solution was proposed not a solution that could give the time evolution for a given time interval. We think that, in general, the applications should present the time evolution of the studied phenomenon. In particular, in the study of a mechanical system where usually the lifetime of the studied apparatus is shorter than 20 , or at most 30 years, the study of the evolution in time of the mechanical system should be by far more important. Furthermore, in the chapter on advanced renewal theory of the Tijms book at page 326, the following is written:

"in many applied probability problems, asymptotic expansions provide a simple alternative to computationally intractable solutions."

We would outline that, in this paper, it is shown that the numerical solution of alternating compound renewal can be obtained in a simple way although the process is a strong generalization of the simple renewal process.

Only the paper Zacks [6] presented an application of alternating compound processes in telegraph problem, but in the particular framework of the Poisson processes not in the general environment of the renewal processes.

In Alvarez [15], a theoretical paper on the alternating renewal processes, in which how to solve the evolution equation analytically but always in a Poisson environment is shown, was presented. The possibility to apply to an engineering problem this tool was also described. But without it any example of this application was not given.

Another paper, Vlasiou [16], gives a very short presentation of alternating compound processes asserting that the total rewards earned are equal to the percentage of the time spent in the UP state with respect to the total time $t$. This result holds in very particular cases and not in a general framework where the rewards, for example, are given by sum of money.

Furthermore, to authors' knowledge, and in any database consulted, never was a paper presented where the costs and the revenues for each period of the time horizon were calculated.

In the previous paper (D'Amico et al. [11]), the authors proposed the application of a discrete time of the simple alternating renewal process in disability insurance, where the rewards were not considered.

The main purpose of this paper is to show how it is possible to apply discrete time alternating compound renewal processes in insurance problems. More specifically, the application will be presented in temporary disability insurance problem generalizing the previous results.

The obtained results are general and can be applied in any other field.

The paper will develop in the following way. In the second section, the discrete time alternating processes in a homogeneous environment will be presented recalling some results obtained in D'Amico et al. [11]. Furthermore, in that paper, the discrete time approach was justified by discussing the strict relation between continuous and discrete time alternating renewal processes. This relation was proved adapting the results obtained in Corradi et al. [17] for homogeneous semiMarkov processes.

In Section 3, the discrete time compound renewal processes will be reported.

In Section 4, the discrete time alternating compound renewal model for the calculation of the mean values (MV), in nondiscounted case, and the mean present values (MPV), in discounted case, is presented. In this way, all the total rewards paid by the insurers for the premiums and by the insurance company for the reported claims in temporary disability insurance will be calculated. In Section 5, examples of the application of the model will be presented. In the last section, some concluding remark will be given. [14].

In this paper, we will follow the notation given in Beichelt

\section{Discrete Time Homogeneous Alternating Processes}

In renewal theory, usually, it is supposed that renewals start as soon as they happen. In real world, it is possible that this condition is not satisfied; that is, renewals can start after a nonnegligible random time. It is possible to take into account this phenomenon, defining a renewal process in which the renewal time after the failure is assumed being an integer nonnegative random variable.

Definition 1. Let $\left\{Y_{1}, Y_{2}, \ldots\right\}$ and $\left\{Z_{1}, Z_{2}, \ldots\right\}$ be two random variables (one will denote r.vs. in this way in singular or plural case) that are supposed to be two independent sequences of independent nonnegative r.v. In this way, the sequence of two-dimensional random vectors $\mathbf{X}=$ $\left\{\left(Y_{1}, Z_{1}\right),\left(Y_{2}, Z_{2}\right), \ldots,\left(Y_{k}, Z_{k}\right), \ldots\right\} Y_{i}, Z_{i} \in \mathbb{N}$ is defined to be 


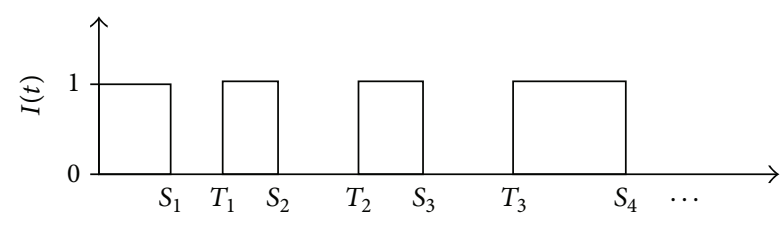

Figure 1: A trajectory of r.v. $I(t)$.

a discrete time alternating renewal process. $Y_{i}$ denotes the $i$ th working period and $Z_{i}$ denotes the $i$ th nonworking period.

It is posed that at time $0^{+}$the system is working and this is the reason why $Y_{i}$ precedes $Z_{i}$.

Remark 2. It is important to outline that the working state depends on the application. In a reliability of an engineering system, then UP state corresponds to the working of the system. In a temporary disability insurance contract, then for the insurance, the UP state is the absence of disability.

The two integer random variables,

$$
\begin{aligned}
& S_{1}=Y_{1}, \\
& S_{n}=\sum_{i=1}^{n-1}\left(Y_{i}+Z_{i}\right)+Y_{n}, \quad n=2,3, \ldots ; S_{n} \in \mathbb{N}, \\
& T_{n}=\sum_{i=1}^{n}\left(Y_{i}+Z_{i}\right), \quad n=1,2, \ldots ; T_{n} \in \mathbb{N},
\end{aligned}
$$

represent the times at which the failures happen and the renewed system starts working, respectively.

From (1), it results in

$$
\begin{aligned}
S_{n} & =T_{n-1}+Y_{n} ; \\
T_{n} & =S_{n}+Z_{n} ; \\
E\left[T_{n}\right] & =n E[Y]+n E[Z] ; \\
E\left[S_{n}\right] & =n E[Y]+(n-1) E[Z] .
\end{aligned}
$$

Now, it is possible to define a state system indicator variable, more precisely,

$$
I(t)= \begin{cases}0 & \text { if } t \in\left[S_{n}, T_{n}\right) \\ 1 & \text { if } t \in\left(\left(0, S_{1}\right) \vee\left[T_{n}, S_{n+1}\right)\right)\end{cases}
$$

In Figure 1, a trajectory of the r.v. (3) is reported.

$Y_{i}$ are i.i.d. r.v. and $Z_{i}$ too. The distribution functions (d.f.) $F_{Y}(y)=P(Y \leq y)$ and $F_{Z}(z)=P(Z \leq z)$ are, respectively, d.f. of $Y_{i}$ and $Z_{i}$.
$N_{Y}(t)$ and $N_{Z}(t)$ are the random numbers of failures and renewals that happened in $(0, t]$, respectively. Given that $S_{n}$ and $T_{n}$ are sums of independent r.v., it results in

$$
\begin{aligned}
F_{S_{n}}(t) & =P\left(S_{n} \leq t\right)=P\left(N_{1}(t) \geq n\right) \\
& =F_{Y} *\left(F_{Z} * F_{Y}\right)^{(n-1)}(t), \\
F_{T_{n}}(t) & =P\left(T_{n} \leq t\right)=P\left(N_{2}(t) \geq n\right) \\
& =\left(F_{Y} * F_{Z}\right)^{(n)}(t) .
\end{aligned}
$$

Remark 3. From now on, we will denote the cumulative distribution functions $(\mathrm{CDF}) F_{Y} * F_{Z}$ by $F_{Y Z}$ and its probability distribution by $f_{Y Z}$ :

(i) $H_{Y}(t)$ and $H_{Z}(t)$ represent the renewal functions of the discrete time alternating renewal process; $H_{Y}(t)$ can be seen as a discrete time delayed renewal process in which the first waiting time d.f. is distributed as $Y$ and the other waiting time d.f. are distributed as $Y+Z$.

In the same way, $H_{Z}(t)$ can be seen as an ordinary discrete time renewal process whose waiting time d.f. are distributed as $Y+Z$.

Proposition 4. It results in the fact that

$$
\begin{array}{r}
E\left(N_{Z}(t)\right)=H_{Z}(t)=\sum_{n=1}^{\infty} F_{Y Z}{ }^{(n)}(t)=F_{Y Z}(t)+H_{Z}(t), \\
E\left(N_{Y}(t)\right)=H_{Y}(t)=F_{Y}(t)+\left(F_{Y, Z}\right) * H_{Y}(t), \\
t=1, \ldots, T .
\end{array}
$$

The proofs of (5) are well known (see Beichelt [14]).

Remark 5. In renewal processes, the time scale can be months, years, or other time intervals depending on the problem that should be studied. In the alternating processes, the time scale length of the $Y$ and $Z$ periods and their means $E(Y)$ and $E(Z)$ are of fundamental relevance. Usually, these mean values do not correspond to the time scale interval or to its multiple.

Remark 6. As proved in Janssen and Manca [18], the discretization of the continuous time homogeneous renewal processes by means of the simplest generalized Newton Cotes formula (rectangle formula) gives the discrete time renewal process. In the same time, starting from the ordinary discrete time renewal process with $h$ as discretization step, it is possible with $h \rightarrow 0$ to obtain the corresponding continuous time renewal process. These properties allow us to ignore the continuous time environment that can be analytically solved only in particular cases and in a very tortuous way (see Adekambi and Mamane [10]). In the most cases, it must be solved numerically which means discretizing the model. We think that the best way is working directly in a discrete time environment. In D'Amico et al. [11], the results given in 
Janssen and Manca [18] were generalized to the alternating renewal process:

$$
\begin{aligned}
& H_{Z}(t)=F_{Y Z}(t)+H_{Z} * F_{Y Z}(t), \\
& H_{Y}(t)=F_{Y}(t)+H_{Y} * F_{Y Z}(t) .
\end{aligned}
$$

Remark 7. The difference between the renewal function of the ordinary renewal process and that of delayed renewal process is given by the known terms of the two integral equations.

$$
\left[\begin{array}{ccccc}
1 & 0 & 0 & \cdots & 0 \\
-v_{Y Z}(1) & 1 & 0 & \cdots & 0 \\
-v_{Y Z}(2) & -v_{Y Z}(1) & 1 & \cdots & 0 \\
\vdots & \vdots & \vdots & \ddots & \vdots \\
-v_{Y Z}(k-2) & -v_{Y Z}(k-3) & -v_{Y Z}(k-4) & \cdots & 1 \\
-v_{Y Z}(k-1) & -v_{Y Z}(k-2) & -v_{Y Z}(k-3) & \cdots & -v_{Y Z}(1) \\
-v_{Y Z}(k) & -v_{Y Z}(k-1) & -v_{Y Z}(k-2) & \cdots & -v_{Y Z}(2) \\
\vdots & \vdots & \vdots & \ddots & \vdots
\end{array}\right.
$$

Remark 8. The system and related matrix form of the $H_{Z}(t)$ evolution equation have the same structure; the only difference is given by the known terms that are $F_{Y Z}(t)$.

\section{Discrete Time Homogeneous Compound Renewal Processes}

Generally speaking, the compound renewal processes are a class of stochastic processes. Indeed, depending on the hypotheses that we need for the model construction, we will get different evolution equations.

Definition 9. Let $\left\{\left(T_{1}, M_{1}\right),\left(T_{2}, M_{2}\right), \ldots\right\}$ be a random marked point process (see Beichelt [14]), where $\left\{T_{1}, T_{2}, \ldots\right\}$ is the sequence of renewal times of a renewal process $\mathbf{X}=\left\{X_{1}, X_{2}, \ldots\right\}$, and let $\{N(t), t \geq 0\}$ be the corresponding renewal counting process and $H(t)=E(N(t))$. Furthermore, $\{C(t), t \geq 0\}$ in nondiscounted and discounted case, respectively, defined by

$$
C(t)= \begin{cases}\sum_{i=1}^{N(t)} M_{i} & \text { if } t \geq 1 \\ 0 & \text { if } t=0\end{cases}
$$

The system that can solve the evolution equation of $H_{Y}(t)$ is reported in the following relation:

$$
\begin{gathered}
H_{Y}(t)-\sum_{\tau=1}^{t} H_{Y}(t-\tau) v_{Y Z}(\tau)=F_{Y}(t), \quad t=1, \ldots, T, \\
H_{Y}(1)=F_{Y}(1), \\
H_{Y}(2)-H_{Y}(1) v_{Y Z}(1)=F_{Y}(2), \\
H_{Y}(3)-H_{Y}(2) v_{Y Z}(1)-H_{Y}(1) v_{Y Z}(2)=F_{Y}(3),
\end{gathered}
$$

where $v_{Y Z}(\tau)=F_{Y Z}(\tau)-F_{Y Z}(\tau-1)$

System (8) in matrix form can be written in the following way:

$$
\left.\begin{array}{rrr}
0 & 0 & \cdots \\
0 & 0 & \cdots \\
0 & 0 & \cdots \\
\vdots & \vdots & \ddots \\
0 & 0 & \cdots \\
1 & 0 & \cdots \\
-v_{Y Z}(1) & 1 & \cdots \\
\vdots & \vdots & \ddots
\end{array}\right] *\left[\begin{array}{c}
H_{Y}(1) \\
H_{Y}(2) \\
H_{Y}(3) \\
\vdots \\
H_{Y}(k-1) \\
H_{Y}(k) \\
H_{Y}(k+1) \\
\vdots
\end{array}\right]=\left[\begin{array}{c}
F_{Y}(1) \\
F_{Y}(2) \\
F_{Y}(3) \\
\vdots \\
F_{Y}(k-1) \\
F_{Y}(k) \\
F_{Y}(k+1) \\
\vdots
\end{array}\right] .
$$

$$
C(t)= \begin{cases}\sum_{i=1}^{N(t)} M_{i} e^{-\delta i} & \text { if } t \geq 1 \\ 0 & \text { if } t=0\end{cases}
$$

is a compound (reward) renewal process and $C(t)$ is called a compound (reward) random variable. $V(t)=E(C(t))$ represents the mean total rewards that were given and/or received in time $t$.

Remark 10. The two sequences $\mathbf{M}=\left\{M_{1}, M_{2}, \ldots\right\}$ and $\mathbf{X}=$ $\left\{X_{1}, X_{2}, \ldots\right\}$ are independent of each other and are n.n.i.i.d. But $M_{i}$ and $X_{j}$ may depend on each other if $i=j$.

Remark 11. The two sequences $\mathbf{M}$ and $\mathbf{X}$ are identically distributed as $M$ and $X$. This hypothesis depends on the homogeneity environment; if a sequence of r.v. is not i.i.d., it cannot be homogeneous (see Gismondi et al. [5]). Under these conditions, we suppose that each element of them will be constant in the time.

The next step is the calculation of the means $E(X)$ and $E(M)$. Regarding the first, there are no problems because it is the mean of interarrival times. Instead, the second 
mean is strictly connected to the evolution equation of the process. In this paper, we will not present all the different evolution equations but only the most general homogeneous nondiscounted case with constant rewards and the related discounted case with deterministic constant rate of interest.

$E(M)$ represents the mean rewards that are given or received during one interarrival time.

In this period, we can have rate reward and impulse reward. The first is an annuity that is paid at each unitary time interval of length $\tau$ and we suppose that $E(X)=k \tau+\tau_{1}$, where $0 \leq \tau_{1}<\tau$. The second is a reward that will be paid if some random event happens. In this paper, we will suppose that the rate rewards are paid at the beginning of each time interval.

In discounted case, $r$ is the related interest rate.

The impulse reward can happen in a period in which it can be paid at the beginning or at the end of the period. We will suppose that it is paid at the end. With the symbols $\psi$ and $\gamma$, we denote the values of one rate reward and one impulse reward. In the following, we report the values of $E(M)$ in nondiscounted and discounted cases, respectively:

$$
\begin{aligned}
E(M) & =\psi\left(k+\frac{\tau_{1}}{\tau}\right)+\gamma, \\
E(M) & =\psi\left(\dot{a}_{k \mid r}+\frac{\tau_{1}}{\tau} e^{-\delta \vartheta}\right)+\gamma e^{-\delta\left(k+\tau_{1} / \tau\right)} ; \\
\delta & =\log (1+r) ; \\
\vartheta & = \begin{cases}k+\frac{\tau_{1}}{\tau} & \text { if immediate } \\
k & \text { if due, }\end{cases} \\
\dot{a}_{k \mid r} & =\frac{1-(1+r)^{-k}}{\rho}, \\
\rho & = \begin{cases}r & \text { if immediate } \\
\frac{r}{1+r} & \text { if due. }\end{cases}
\end{aligned}
$$

Remark 12. In nondiscounted case, the time is not relevant; this fact implies that the Wald identities hold.

In discounted case, the value $E(M)$ is calculated at the beginning of the period; $\gamma$ can be paid at the beginning or at the end of each period; we suppose that it is paid at the end. $E(M)$ is always the same for each period, but it should be discounted at time 0 . Its value changes in function of the time in which it is paid. In relation (6), $\dot{a}_{\bar{k}}{ }_{r}$ and $\vartheta$ depend on the time of installment payments. Furthermore, in this case, it is not possible to apply the Wald identities but one of its simple generalizations.

Then, $H(t)$ represents the mean number of renewals that happens at times $0, E(X), 2 E(X), \ldots, q E(X)$ with the first $q$ installments equal to $E(M)$ and the last, paid at time $q E(X)$.

In the nondiscounted, if we set $V(t)=E(C(t))$ it results:

$$
V(t)=H(t) E(M) .
$$

In discounted case, $E(M)$ represents an installment of a due annuity.
Proposition 13. The evolution equation in the discounted case is given by

$$
\begin{aligned}
& V(t)=E(M) \\
& \cdot\left(\ddot{a} \overline{\lfloor H(t)\rfloor r_{E(X)}}+(H(t)-\lfloor H(t)\rfloor) \cdot e^{-\delta\lfloor H(t)\rfloor}\right),
\end{aligned}
$$

where

$$
\begin{aligned}
& r_{E(X)}=(1+r)^{E(X)}-1 ; \\
& \ddot{a} \overline{\lfloor H(t)\rfloor r_{E(X)}}= \begin{cases}\frac{1-e^{-\delta_{E(X)}\lfloor H(t)\rfloor}}{d_{E(X)}} & \text { if } H(t) \geq 1 \\
0 & \text { if } H(t)<1 ;\end{cases} \\
& d_{E(X)}=\frac{r_{E(X)}}{1+r_{E(X)}} ; \\
& \delta_{E(X)}=\log \left(1+r_{E(X)}\right) \text {; } \\
& \lfloor b\rfloor=\text { floor }(b) \text {. }
\end{aligned}
$$

Proof. $E(M)$ is discounted at the beginning of each renewal period. It is considered as a constant installment of a due annuity. $H(t) \in \mathbb{R}$ is the mean number of renewals that happen from time 0 to time $t$. If $\lfloor H(t)\rfloor=H(t)$, then the second term of the sum in (13) is equal to zero. In the other case,

$$
E(M) \cdot(H(t)-\lfloor H(t)\rfloor)
$$

represents the value of the last installment that is smaller than $E(M)$ and should be discounted for the time period $\lfloor H(t)\rfloor$ because it is supposed that these mean installments are paid at the beginning of each period.

Remark 14. In (14), some simple well-known financial equalities are given.

\section{Discrete Time Homogeneous Alternating Compound Processes}

Definition 15. Let $\overline{\mathbf{M}}=\left\{M_{1,0}, M_{1,1}, M_{2,1}, M_{2,2}, M_{3,2}, M_{3,3}\right.$, $\ldots\}$ and $\mathbf{A}=\left\{\left(Y_{1}, Z_{1}\right),\left(Y_{2}, Z_{2}\right), \ldots\right\}$ be, respectively, a sequence of n.n.i.i.d., d.t., and r.v. and a sequence of twodimensional random vectors, that is, a homogenous alternating renewal process. Moreover, $N_{Y}(t)$ and $N_{Z}(t), t \geq 0$, are the random numbers of failures and renewals that happened in $(0, t]$, respectively. Then, the stochastic process is given by the couple $\left\{\left(C_{Y}(t), C_{Z}(t)\right), t \geq 0\right\}$, where in nondiscounted and in discounted cases, respectively, it results in

$$
C_{Y}(t)= \begin{cases}\sum_{i=1}^{N_{Y}(t)} M_{i, i-1} & \text { if } t \geq 1 \\ 0 & \text { if } t=0\end{cases}
$$




$$
\begin{aligned}
& C_{Z}(t)= \begin{cases}\sum_{i=1}^{N_{Z}(t)} M_{i, i} & \text { if } t \geq 1 \\
0 & \text { if } t=0,\end{cases} \\
& C_{Y}(t)= \begin{cases}\sum_{i=1}^{N_{Y}(t)} M_{i, i-1} \cdot e^{-\delta_{Y Z}(i-1)} & \text { if } t \geq 1 \\
0 & \text { if } t=0 ;\end{cases} \\
& C_{Z}(t)= \begin{cases}\sum_{i=1}^{N_{Z}(t)} M_{i, i} \cdot e^{-\left(\delta_{Y}+\delta_{Y Z}(i-1)\right)} & \text { if } t \geq 1 \\
0 & \text { if } t=0 .\end{cases}
\end{aligned}
$$

It is called an alternating compound (reward) renewal process. $C_{Y}(t)$ and $C_{Z}(t)$ are called the alternating compound random variables.

$V_{Y}(t)=E\left(C_{Y}(t)\right)$ and $V_{Z}(t)=E\left(C_{Z}(t)\right)$ represent the mean total rewards that were given and/or received during failure or renewal cycles in a time $t$, respectively.

Remark 16. The two sequences $\overline{\mathbf{M}}$ and $\mathbf{A}$ are independent and $M_{i, i-1}$ corresponds to $Y_{i}$ and $M_{i, i}$ corresponds to $Z_{i}$.

Remark 17. The r.v. sequences $\mathbf{M}_{Y}=\left(M_{n, n-1}\right)_{n \geq 1}, \mathbf{M}_{Z}=$ $\left(M_{n, n}\right)_{n \geq 1}, \mathbf{Y}=\left(Y_{n}\right)_{n \geq 1}, \mathbf{Z}=\left(Z_{n}\right)_{n \geq 1}$ are identically distributed because of homogeneity. Also, in this case, each element of each r.v. sequence is constant in the time. We can find their means $E(Y), E(Z), E\left(M_{Y}\right)$, and $E\left(M_{Z}\right)$.

$E(Y)$ and $E(Z)$ represent the mean of interarrival time of $Y$ and $Z$, respectively.

$E\left(M_{Y}\right)$ represents the mean rewards that are given or received during one interarrival time of $Y$. Similarly, $E\left(M_{Z}\right)$ represents the mean rewards that are given or received during one interarrival time of $Z$.

As for the renewal compound, we can have rate and impulse reward that we will suppose constant in the time. The corresponding annuities will be paid at each time interval of length $\tau_{Y}$ and $\tau_{Z}$, respectively. We suppose that $E(Y)=$ $k_{Y} \tau_{Y}+\tau_{1}$, where $0 \leq \tau_{1}<\tau_{Y}$, and $E(Z)=k_{Z} \tau_{Z}+\tau_{2}$, where $0 \leq \tau_{2}<\tau_{Z}$ and $k_{Y}, k_{Z} \in \mathbb{N}$. The impulse rewards $\gamma_{Y}$ and $\gamma_{Z}$ will be paid if some random events will happen.

We present the nondiscounted and discounted evolution equations of the alternating compound process. Furthermore, we denote by $\psi_{Y}$ and $\psi_{Z}$ the constant rate rewards of $\mathbf{Y}$ and $\mathbf{Z}$, respectively. In discounted case, $r$ is the fixed interest rate for a time interval.

Remark 18. We could consider the $\mathbf{Y}$ process discounted and the $\mathbf{Z}$ processes nondiscounted or vice versa but we would consider only two cases taking into account developing these aspects in a future paper.

In (17) and (18), the nondiscounted cases, and in (19) and (20), the discounted cases, $E\left(M_{Y}\right)$ and $E\left(M_{Z}\right)$ are reported.
They represent the mean of rewards in $E(Y)$ and $E(Z)$, respectively:

$$
\begin{aligned}
E\left(M_{Y}\right) & =\psi_{Y}\left(k_{Y}+\frac{\tau_{1}}{\tau_{Y}}\right)+\gamma_{Y}, \\
E\left(M_{Z}\right) & =\psi_{Z}\left(k_{Z}+\frac{\tau_{2}}{\tau_{Z}}\right)+\gamma_{Z}, \\
E\left(M_{Y}\right) & =\psi_{Y}\left(\dot{a}_{\left.k_{Y}\right|^{r}}+\frac{\tau_{1}}{\tau_{Y}} e^{-\delta \vartheta_{Y}}\right)+\gamma_{Y} e^{-\delta\left(k_{Y}+\tau_{1} / \tau_{Y}\right)}, \\
\vartheta_{Y} & = \begin{cases}k_{Y}+\frac{\tau_{1}}{\tau_{Y}} & \text { if immediate } \\
k_{Y} & \text { if due, }\end{cases} \\
\dot{a}_{\left.k_{Y}\right|^{r}} & =\frac{1-(1+r)^{-k_{Y}}}{\rho}, \\
E\left(M_{Z}\right) & =\psi_{Z}\left(\dot{a}_{\left.k_{Z}\right|^{r}}+\frac{\tau_{1}}{\tau_{Z}} e^{-\delta \vartheta_{Z}}\right)+\gamma_{Z} e^{-\delta\left(k_{Z}+\tau_{2} / \tau_{Z}\right)}, \\
\dot{a}_{\left.k_{Z}\right|^{r}} & =\frac{1-(1+r)^{-k_{Z}}}{\rho}, \\
\vartheta_{Z} & = \begin{cases}k_{Z}+\frac{\tau_{2}}{\tau_{Z}} & \text { if immediate } \\
k_{Z} & \text { if due, }\end{cases}
\end{aligned}
$$

where $\rho$ is calculated as in (6). Furthermore, it results in the fact that $\dot{a}_{0, r}=0$.

Remark 19. Wald's identity holds in the nondiscounted case. As for the compound renewal in discounted case, the values $E\left(M_{Y}\right)$ and $E\left(M_{Z}\right)$ are discounted at the beginning of each renewal period. It is supposed that $\gamma_{Y}$ and $\gamma_{Z}$ are paid at the end of each period. Furthermore, it is to outline that $\mathbf{Y}$ can be considered as a delayed renewal process (see Beichelt [14]).

Remark 20. In discounted case, $E\left(M_{Y}\right)$ and $E\left(M_{Z}\right)$ represent instalments of due annuities.

In alternating renewal processes, the time $t$, taking into account the mean times $E(Y)$ and $E(Z)$, can have two different situations that are mutually exclusive; that is,

$$
\begin{aligned}
& t_{Z}=E(Y)+q_{Z}(E(Z)+E(Y))+\lambda_{Z} \\
& \quad \text { where } q_{Z} \in \mathbb{N}_{0}, 0 \leq \lambda_{Z}<E(Z), \\
& t_{Y}=q_{Y}(E(Z)+E(Y))+\lambda_{Y} \\
& \text { where } q_{Y} \in \mathbb{N}_{0}, 0 \leq \lambda_{Y}<E(Y) .
\end{aligned}
$$

Remark 21. We suppose that the rewards corresponding to $Y$ will be given only in the times $t_{Y}$ and the ones of $Z$ will be given in $t_{Z}$. in

In nondiscounted case, Wald's identity holds and it results

$$
\begin{aligned}
& V_{Y}\left(t_{Y}\right)=E\left(M_{Y}\right) \cdot H_{Y}\left(t_{Y}\right) ; \\
& V_{Z}\left(t_{Z}\right)=E\left(M_{Z}\right) \cdot H_{Z}\left(t_{Z}\right) .
\end{aligned}
$$




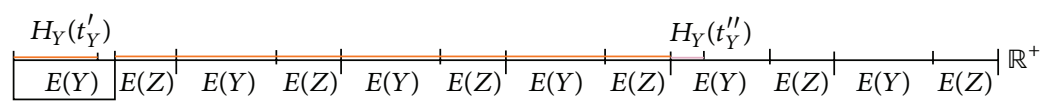

FIgURE 2: Time axis representing case (25).

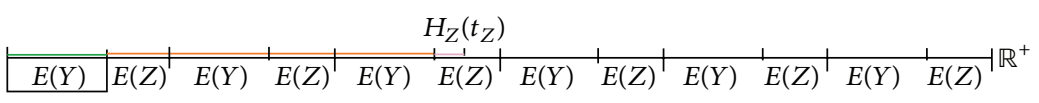

FIgURE 3: Time axis representing case (26). The deferred period is in green.

Remark 22. Regarding the discounted case, it is to point out that $E\left(M_{Y}\right)$ is discounted at the beginning of the mean period $E(Y)$ and, similarly, $E\left(M_{Z}\right)$ at the beginning of the mean period $E(Z)$. For this reason, it is necessary to calculate the following equivalent interest rates:

$$
\begin{aligned}
r_{E(Z)} & =(1+r)^{E(Z)}-1, \\
r_{E(Y)} & =(1+r)^{E(Y)}-1, \\
r_{E(Z)+E(Y)} & =(1+r)^{E(Z)+E(Y)}-1
\end{aligned}
$$

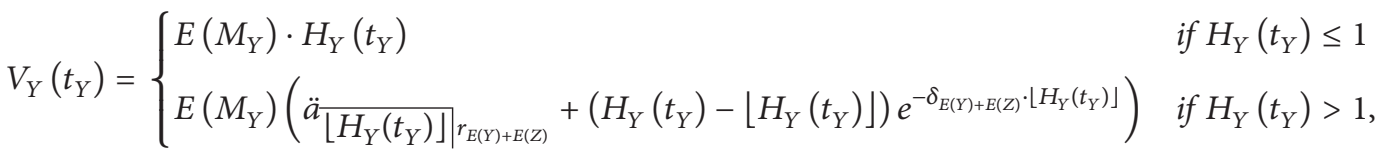

$$
\begin{aligned}
& V_{Z}\left(t_{Z}\right)=e^{-\delta_{E(Y)}} \cdot\left(\ddot{a} \overline{\left\lfloor H_{Z}\left(t_{Z}\right)\right\rfloor \mid r_{E(Z)+E(Y)}}+\left(H_{Z}\left(t_{Z}\right)-\left\lfloor H_{Z}\left(t_{Z}\right)\right\rfloor\right) e^{-\delta_{E(Y)+E(Z)} \cdot\left\lfloor H_{Z}\left(t_{Z}\right)\right\rfloor}\right) E\left(M_{Z}\right) .
\end{aligned}
$$

Proof. We will explain first relation (25). We have two different cases. In the first, we have $H_{Y} \leq 1$, the mean total rewards are paid for a period $H_{Y}\left(t_{Y}\right) \leq E(Y)$, and $E\left(M_{Y}\right)$ is already discounted at time 0 and paid for time units less than or equal to $E(Y)$. In the second case, the mean total rewards can be divided into two parts. In the first

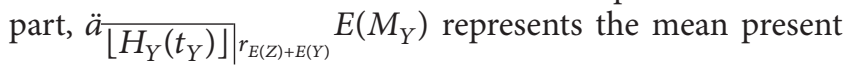
value of the $\left\lfloor H_{Y}\left(t_{Y}\right)\right\rfloor$ mean instalments that were totally paid within the time $t_{Y}$.

The second represents the part of the last instalment that was paid. This part is given by

$$
H_{Y}\left(t_{Y}\right)-\left\lfloor H_{Y}\left(t_{Y}\right)\right\rfloor \text {. }
$$

This part should be discounted for $\left\lfloor H_{Y}\left(t_{Y}\right)\right\rfloor$ periods of length $E(Y)+E(Z)$, given that the mean instalments are discounted at the beginning of the last partial period of length $E(Y)$.

Relation (26) is similar to the second part of (25); the only difference is that in this case it is necessary to discount the values by a supplemental period of length $E(Y)$.

The two cases of (25) are shown in Figure 2. In this picture, $H_{Y}\left(t_{Y}^{\prime}\right)$ represents the first case corresponding to the unit of time. Furthermore, $E\left(M_{Y}\right) \cdot H_{Y}^{\prime} / E(Y)$ represents the reward paid in the time $H_{Y}\left(t_{Y}^{\prime}\right)$.
$H_{Y}\left(t_{Y}^{\prime \prime}\right)$ corresponds to the time interval of the second case of (25). It is formed by four time periods of length $E(Y)+E(Z)$ plus $H_{Y}\left(t_{Y}^{\prime \prime}\right)-\left\lfloor H_{Y}\left(t_{Y}^{\prime \prime}\right)\right\rfloor$.

Figure 3 shows a time axis of relation (26). From the figure, it is simple to understand why the results must be discounted, further, for a time of length $E(Y)$.

Remark 24. Taking into account the alternating model, we suppose that when the system is in $E(Z)$, nothing will be paid to the people that are paid in $E(Y)$ and vice versa. We also remark that it is also possible to consider these payments.

In Figure 4 is reported a trajectory of an alternating compound renewal process.

\section{The Algorithm Description}

The algorithm is long but not tortuous; in this section, we will describe the main step without entering in detail (see Algorithm 1).

In the last part of Algorithm 1's description is reported the related Mathematica program.

Where aafigYZ[ $[\mathrm{kY}]]$ is the present value of a due unitary annuity in which the unit time is $E(Y)+E(Z)$ and $k Y$ represents the number of instalments, aafigYZY [ [kZ] ] represents the previous unitary annuity deferred of a time 


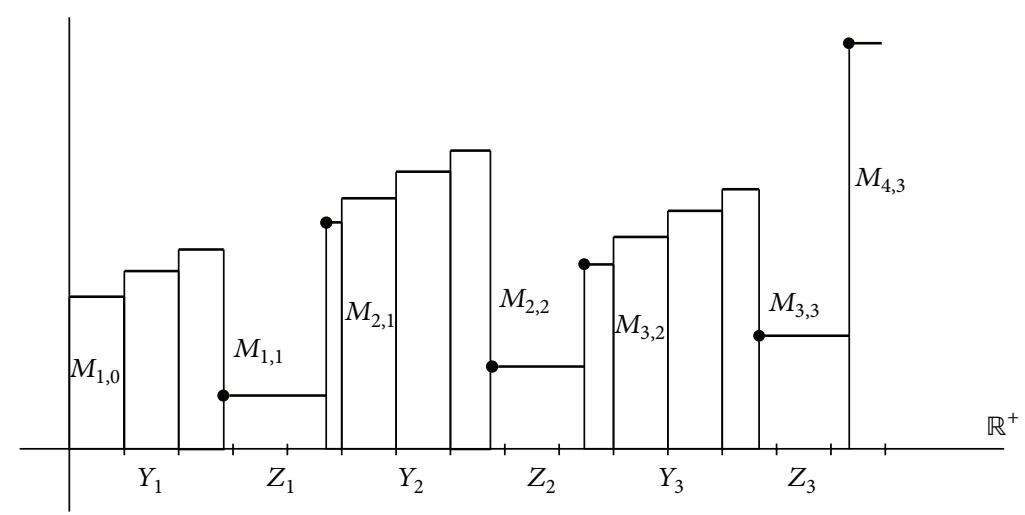

FIGURE 4: A trajectory of a nondiscounted alternating compound renewal process with impulse reward and rate rewards in $Y$ and only impulse rewards in $Z$. The impulse rewards are variable. $M_{j, j-1}$ represent the total value of the $Y_{j}$ rewards and $M_{j, j}$ the total value of the $Z_{j}$ rewards.
(1) Inputs:
(1.1) the interest rate structure (that could be flat, a term structure and stochastic),
(1.2) the rate and impulse rewards (constant or variable),
(1.3) the time unit number of CDF,
(1.4) the CDF.
(2) Construction of the elementary financial data,
(2.1) the construction of discount factors,

(2.2) the construction of due and immediate unitary annuity present value (in the case of constant rewards).

(3) Convolution $F_{Y} * F_{Z}(t)$.

(4) Construction of $H_{Y}$ and $H_{Z}$.

(5) Calculation of

$E(Y), E(Z), E\left(M_{Y}\right), E\left(M_{Z}\right), E(Y+Z)$

(6) Calculation of other financial data,

(6.1) equivalent interest:

(6.1.1) $r E(Y)=(1+r)^{\wedge} E(Y)-1$,

(6.1.2) $r E(Z)=(1+r)^{\wedge} E(Z)-1$,

(6.1.3) $r E(Y) E(Z)=(1+r)^{\wedge} E(Y+Z)-1$.

(6.2) Instantaneous intensity:

(6.2.1) $\mathrm{De}=\log (1+r)$,

(6.2.2) $\operatorname{De} E(Y)=\log (1+r E(Y))$,

(6.2.3) $\operatorname{De} E(Z)=\log (1+r E(Z))$,

(6.2.4) $\operatorname{De} E(Y) E(Z)=\log (1+r E(Y) E(Z))$.

(7) Calculation of $V_{Y}$ and $V_{Z}$

$\mathrm{VY}=\mathrm{Table}[0.0,\{\mathbf{i}, 1,\{$ nannpYZ $\}]$;

$\mathrm{VZ}=\mathrm{Table}[0.0,\{i, 1,\{$ nannpYZ $\}$;

For $[t=1, t<=$ nannpYZ, $t++$,

$\mathrm{kY}=\mathrm{Floor}[\mathrm{HY}[[\mathrm{t}]]]$;

$\mathrm{hY}=\mathrm{HY}[[\mathrm{t}]]-\mathrm{kY}$;

If $[k Y>0$,

$\mathrm{VY}[[\mathrm{t}]]+=\mathrm{N}[$ aafigYZ $[\mathrm{kY}]] * \mathrm{EMY}, 64]$;

] ;

$\mathrm{VY}[[\mathrm{t}]]+=\mathrm{N}[\mathrm{hY} * \operatorname{EMY} * \operatorname{Exp}[-\operatorname{DeEYEZ} * \mathrm{kY}], 64]$

$\mathrm{kZ}=\mathrm{Floor}[\mathrm{HZ}[\mathrm{t}]]]$;

$\mathrm{hZ}=\mathrm{HZ}[[\mathrm{t}]]-\mathrm{kZ}$;

If $[\mathrm{kZ}>0$,

$\operatorname{VZ}[[\mathrm{t}]]+=\mathrm{N}[$ aafigYZY $[\mathrm{kZ}]] * \mathrm{EMZ}, 64]$;

] ;

$\mathrm{VZ}[[\mathrm{t}]]+=\mathrm{N}[\mathrm{EMZ} * \mathrm{hZ} * \operatorname{Exp}[-\mathrm{DeEY}-\operatorname{DeEYEZ} * \mathrm{kZ}], 64]$;

]; 

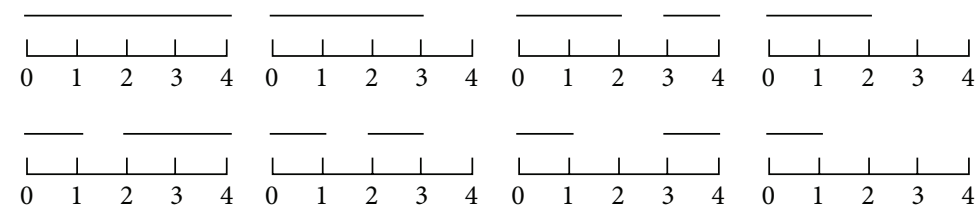

FIGURE 5: Possible trajectories in four years with starting state healthy.

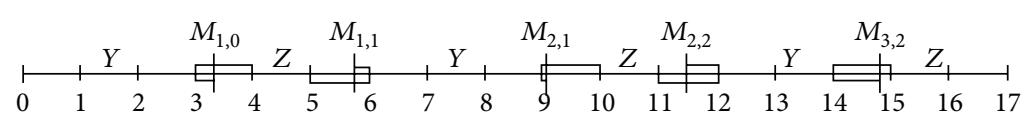

FIGURE 6: Mean financial evolution of an alternating compound renewal process. The tall segments divide the $Y$ periods from the $Z$ periods, the short the years. It is supposed that the durations of each period $Y$ and $Z$ are $E(Y)$ and $E(Z)$, respectively. Only the last period will have different duration depending on the length of the contract. The closed boxes denote the rate rewards paid for a period less than one time unit. The length $M_{i, i-1}-$ Floor $\left(M_{i, i-1}\right)$ represents the last part of the $i$ th $Y$ (see, e.g., the length of the segment that goes from 3 to $M_{1,0}$ ). The length Ceiling $\left(M_{i, i}\right)-M_{i, i}$ shows the first part of the $i+1$ th $Y$ (see, e.g., the length of the segment that goes from $M_{2,2}$ to 12). The one side open boxes represent the $Z$ rate rewards paid for a period less than one time unit. The lengths Ceiling $\left(M_{i, i-1}\right)-M_{i, i-1}$ and $M_{i, i}-$ Floor $\left(M_{i, i}\right)$ give, respectively, the first and the last parts of the $i$ th $Z$-period.

$E(Y) . \mathrm{N}[\ldots, 64]$ means that the calculations are done with 64 digits.

Remark 25. $E(Y), E(Z)$ are the reward mean time of $Y$ and $Z$, respectively. $E\left(M_{Y}\right), E\left(M_{Z}\right)$ are the mean reward paid in $E(Y)$ and in $E(Z)$; they are discounted at the beginning of the mean periods.

\section{The Temporary Disability Insurance Studied by a D. T. Alternating Compound Renewal Model}

6.1. The Insurance Problem. In this section, we apply the discrete time homogeneous alternating compound renewal process to the temporary disability insurance contract.

In this case, the insured can be temporarily disabled, that is, cannot work, or be healthy. It is clear that the insurance company will never accept at the beginning of the contract a disabled person. For the insurance company, the healthy state is the "working state" and the illness is the not working state.

The contract has a yearly premium payment. We are interested to find the present value of the cost of claims that the insurance company should pay to the insured person and the present value of premiums that the company will receive. We can model this insurance contract by a discrete time homogeneous alternating compound renewal model.

In Figure 5, the trajectories that can be obtained in 4 time periods with starting state healthy are reported. The first period is healthy because an insurance company will not accept any insured that at the beginning of the contract is ill. For this reason, the only possible trajectories are $2^{3}=8$.

6.2. Applicative Examples. We did not have data that could permit a precise construction of input for our model. However, we decided to construct four examples that will show how our model works.
TABLE $1: Y$ and $Z$ CDF

\begin{tabular}{lcc}
\hline Time & $\begin{array}{c}\text { Input data } \\
\text { CDF_Y }\end{array}$ & CDF_Z \\
\hline 0 & 0 & 0 \\
1 & 0.014476 & 0.144349 \\
2 & 0.019221 & 0.515605 \\
3 & 0.025497 & 0.806072 \\
4 & 0.083142 & 0.86736 \\
5 & 0.127643 & 0.908418 \\
6 & 0.206742 & 0.939994 \\
7 & 0.245294 & 0.958056 \\
8 & 0.351764 & 0.970789 \\
9 & 0.446537 & 0.981991 \\
10 & 0.537532 & 0.988795 \\
11 & 0.641189 & 0.992272 \\
12 & 0.724329 & 0.994815 \\
13 & 0.819436 & 0.996897 \\
14 & 0.923688 & 0.998432 \\
15 & 1 & 1 \\
\hline
\end{tabular}

In these applications, we suppose that the insurance will not receive impulse rewards and that the mean benefit of the insured is an impulse reward.

Our starting horizon time was 15 years. Taking into account the convolution of the $Y$ and $Z$ random variables, we covered a period of 30 years. In the first two examples, we suppose that the insurance company will get $300 €$ per time unit as mean premium by the insured people received at the beginning of the unit time. The insured will get $3000 €$ as reimbursement per each $Z$ renewal period by the company supposing that it is paid at the end of the period. The yearly interest rate is $3 \%$. The present value of the rate rewards received as premium by the insurance company was 
TABLE 2: Distribution function $F_{Y Z}$.

\begin{tabular}{|c|c|c|c|c|c|c|c|c|c|}
\hline \multicolumn{10}{|c|}{ CDF_YZ } \\
\hline Time & 0 & 1 & 2 & 3 & 4 & 5 & 6 & 7 & 8 \\
\hline Prob. & 0 & 0 & 0.00209 & 0.008149 & 0.015021 & 0.027938 & 0.05847 & 0.10419 & 0.156249 \\
\hline Time & 9 & 10 & 11 & 12 & 13 & 14 & 15 & 16 & 17 \\
\hline Prob. & 0.214468 & 0.287706 & 0.375240 & 0.463810 & 0.554860 & 0.645052 & 0.736492 & 0.830444 & 0.906403 \\
\hline Time & 18 & 19 & 20 & 21 & 22 & 23 & 24 & 25 & 26 \\
\hline Prob. & 0.946832 & 0.964069 & 0.975923 & 0.984026 & 0.989375 & 0.993217 & 0.995827 & 0.997425 & 0.998444 \\
\hline Time & 27 & 28 & 29 & 30 & & & & & \\
\hline Prob. & & & & & & & & & \\
\hline
\end{tabular}

discounted at the beginning of each mean renewal period of length $E(Y)=9.83$ by the present value of a due unitary installment. It was supposed that the other inputs were the CDF of $Y$ and $Z$ that are reported in Table 1.

In Table 2, the d.f. of $F_{Y} * F_{Z}$ is shown.

In Table 3, the mean total number of claims reported within each year $t$ of the considered time horizon is reported.

Remark 26. In Table $3, H_{Y}(1)>0$ instead of $H_{Z}(1)=0$. Indeed, this fact depends on the different known terms of the two mean numbers. It results clear in looking (7) and (8).

6.2.1. I Case. The data given in the previous three tables are common to all the given cases. In this first case, we suppose that the interest rate is $3 \%$. The mean present value is $E\left(M_{Y}\right)=$ $2597.55 €$. The mean benefit paid by the insurance company has a mean renewal period of length $E(Z)=2.94$. The $3000 €$ were discounted at the beginning of the $E(Z)$ period and its mean discounted value is $E\left(M_{Z}\right)=-2750.61 €$. Its value is negative because we are working from the viewpoint of the insurance company.

In Table 4 are reported the results of our first example.

Remark 27. It looks strange that the value of $V_{Y}$ at time 5 is smaller than the one in 4 and the value in row 6 is smaller than both but it depends on the actualization; indeed, before it, they are equal to 228.376, 248.296, and 269.244, respectively.

6.2.2. II Case. In the first case, the mean present value of the insurance company was smaller than the mean present value of the insured. This fact does not happen usually. In this second case, the premium is brought at $350 €$ with a mean present value equal to 3030.48 . The claim value was not changed.

In Table 5 are reported the related values.

6.2.3. III Case. In this case, we suppose that the rate of interest is always 3\%, but we suppose also that there is an increasing of the $1.8 \%$ of the premiums of the company and an increasing of the $1.5 \%$ of the mean claim value.
TABLE 3: Mean number of renewals reported within each year for $Y$ and $Z$.

\begin{tabular}{lcc}
\hline & Mean number of claims & \\
Time & $H_{Y} H_{Y}$ & $H_{Z}$ \\
\hline 0 & 0 & 0 \\
1 & 0.014476 & 0 \\
2 & 0.019221 & 0.00209 \\
3 & 0.025527 & 0.008149 \\
4 & 0.08327 & 0.015026 \\
5 & 0.127912 & 0.027967 \\
6 & 0.20739 & 0.058566 \\
7 & 0.246932 & 0.104423 \\
8 & 0.355123 & 0.156813 \\
9 & 0.452676 & 0.215772 \\
10 & 0.548401 & 0.29037 \\
11 & 0.659883 & 0.380202 \\
12 & 0.754524 & 0.472627 \\
13 & 0.865198 & 0.569899 \\
14 & 0.990609 & 0.669364 \\
15 & 1.094958 & 0.773789 \\
16 & 1.130467 & 0.885427 \\
17 & 1.174013 & 0.984889 \\
18 & 1.225479 & 1.055479 \\
19 & 1.285403 & 1.110094 \\
20 & 1.354001 & 1.166913 \\
21 & 1.430345 & 1.227918 \\
22 & 1.511493 & 1.294302 \\
23 & 1.595246 & 1.366705 \\
24 & 1.681536 & 1.443804 \\
25 & 1.769417 & 1.77759 \\
26 & 1.856478 \\
27 & 1.941151 & 1.862118 \\
28 & 2.023513 & 1.945029 \\
29 & 2.103553 & \\
30 & 2.181583 & \\
\hline & & \\
& &
\end{tabular}

By means of the following relation, it will be possible to calculate the real rate of interest that will be used in the discounting factor:

$$
\frac{1+r}{1+r_{i}}-1
$$

where $r$ is the interest rate and $r_{i}$ the inflation rate. 
TABLE 4: Results of the first case.

\begin{tabular}{|c|c|c|}
\hline Time & $\begin{array}{c}\text { Premium } 300 € \\
V_{Y}\end{array}$ & $\begin{array}{c}\text { Benefit } 3000 € \\
V_{Z}\end{array}$ \\
\hline 0 & 0 & 0 \\
\hline 1 & 37.60217 & 0 \\
\hline 2 & 49.92756 & -4.29789 \\
\hline 3 & 66.30837 & -16.7606 \\
\hline 4 & 216.2974 & -30.9047 \\
\hline 5 & 332.2589 & -57.5234 \\
\hline 6 & 538.7058 & -120.458 \\
\hline 7 & 641.4176 & -214.777 \\
\hline 8 & 922.4504 & -322.535 \\
\hline 9 & 1175.85 & -443.802 \\
\hline 10 & 1424.5 & -597.236 \\
\hline 11 & 1714.081 & -782.003 \\
\hline 12 & 1959.916 & -972.105 \\
\hline 13 & 2247.397 & -1172.17 \\
\hline 14 & 2573.158 & -1376.75 \\
\hline 15 & 2766.663 & -1591.54 \\
\hline 16 & 2829.9 & -1821.15 \\
\hline 17 & 2907.45 & -2025.73 \\
\hline 18 & 2999.106 & -2135.04 \\
\hline 19 & 3105.824 & -2212.06 \\
\hline 20 & 3227.99 & -2292.18 \\
\hline 21 & 3363.95 & -2378.21 \\
\hline 22 & 3508.466 & -2471.82 \\
\hline 23 & 3657.62 & -2573.92 \\
\hline 24 & 3811.294 & -2682.64 \\
\hline 25 & 3967.8 & -2795.93 \\
\hline 26 & 4122.846 & -2913 \\
\hline 27 & 4273.64 & -3032.83 \\
\hline 28 & 4407.152 & -3153.34 \\
\hline 29 & 4504.88 & -3272.53 \\
\hline 30 & 4600.153 & -3389.45 \\
\hline
\end{tabular}

Having two different inflation rates, we will have two different interest rates.

More precisely, the real interest rate of premiums is $1.179 \%$ and of benefits $1.478 \%$. In this case, the mean present value is $E\left(M_{Y}\right)=2802.56 €$ and claim cost -2873.52 . The time evolution of the means of premiums and of claim costs is reported in Table 6.

6.2.4. IV Case. In the third case, the mean of premiums is lower than the benefit mean. As before, we brought the one time period of the premium at $350 €$ obtaining $E\left(M_{Y}\right)=$ $3269.65 €$. The evolution of the premiums and of claim costs of this last case is given in Table 7.

Remark 28. The cases three and four are calculated with two different discount rates. The algorithm described in Section 5
TABLE 5: Results of the second case.

\begin{tabular}{|c|c|c|}
\hline Time & $\begin{array}{c}\text { Premium } 350 € \\
V_{Y}\end{array}$ & $\begin{array}{c}\text { Benefit } 3000 € \\
V_{Z}\end{array}$ \\
\hline 0 & 0 & 0 \\
\hline 1 & 43.869199 & 0 \\
\hline 2 & 58.248817 & -4.29789 \\
\hline 3 & 77.359765 & -16.7606 \\
\hline 4 & 252.34692 & -30.9047 \\
\hline 5 & 387.63538 & -57.5234 \\
\hline 6 & 628.49006 & -120.458 \\
\hline 7 & 748.32055 & -214.777 \\
\hline 8 & 1076.1921 & -322.535 \\
\hline 9 & 1371.8247 & -443.802 \\
\hline 10 & 1661.9161 & -597.236 \\
\hline 11 & 1999.7613 & -782.003 \\
\hline 12 & 2286.5685 & -972.105 \\
\hline 13 & 2621.9626 & -1172.17 \\
\hline 14 & 3002.0174 & -1376.75 \\
\hline 15 & 3227.7734 & -1591.54 \\
\hline 16 & 3301.5503 & -1821.15 \\
\hline 17 & 3392.0256 & -2025.73 \\
\hline 18 & 3498.957 & -2135.04 \\
\hline 19 & 3623.4617 & -2212.06 \\
\hline 20 & 3765.9884 & -2292.18 \\
\hline 21 & 3924.6081 & -2378.21 \\
\hline 22 & 4093.2108 & -2471.82 \\
\hline 23 & 4267.2236 & -2573.92 \\
\hline 24 & 4446.5096 & -2682.64 \\
\hline 25 & 4629.0995 & -2795.93 \\
\hline 26 & 4809.9865 & -2913 \\
\hline 27 & 4985.9136 & -3032.83 \\
\hline 28 & 5141.6778 & -3153.34 \\
\hline 29 & 5255.6936 & -3272.53 \\
\hline 30 & 5366.8456 & -3389.45 \\
\hline
\end{tabular}

changes slightly and we think that it is not necessary to rewrite more or less the same algorithm.

\section{Conclusion}

In this paper, the discrete time alternating compound renewal process, in homogeneous case, was presented. It is to outline that this paper for the first time gives the time evolution of a compound alternating renewal process and despite what was written in literature (see Tijms [13]) it is shown how simple the application of this process is. Usually the papers that apply the alternating process in continuous time have a very huge theoretical part and if there are applications, they are very simple examples. Furthermore, many applications give the asymptotic results but never the time evolution of the process.

To authors' knowledge, the compound alternating processes were never applied in a general framework. This is another strength of this paper that gives the tool for the 
TABLE 6: Third case results.

\begin{tabular}{|c|c|c|}
\hline Time & $\begin{array}{c}\text { Premium } 300 € \\
V_{Y}\end{array}$ & $\begin{array}{c}\text { Benefit } 3000 € \\
V_{Z}\end{array}$ \\
\hline 0 & 0 & 0 \\
\hline 1 & 40.56984881 & 0 \\
\hline 2 & 53.86799282 & -5.19786 \\
\hline 3 & 71.54162948 & -20.2702 \\
\hline 4 & 233.3682117 & -37.3761 \\
\hline 5 & 358.481782 & -69.5687 \\
\hline 6 & 581.2220687 & -145.682 \\
\hline 7 & 692.0402553 & -259.751 \\
\hline 8 & 995.252993 & -390.073 \\
\hline 9 & 1268.651391 & -536.734 \\
\hline 10 & 1536.925406 & -722.296 \\
\hline 11 & 1849.361647 & -945.753 \\
\hline 12 & 2114.598448 & -1175.66 \\
\hline 13 & 2424.767918 & -1417.62 \\
\hline 14 & 2776.239291 & -1665.04 \\
\hline 15 & 3031.697365 & -1924.8 \\
\hline 16 & 3117.381572 & -2202.5 \\
\hline 17 & 3222.459179 & -2449.91 \\
\hline 18 & 3346.648929 & -2601.93 \\
\hline 19 & 3491.248083 & -2714.58 \\
\hline 20 & 3656.77799 & -2831.77 \\
\hline 21 & 3840.998257 & -2957.59 \\
\hline 22 & 4036.812728 & -3094.52 \\
\hline 23 & 4238.910474 & -3243.85 \\
\hline 24 & 4447.132468 & -3402.87 \\
\hline 25 & 4659.191563 & -3568.57 \\
\hline 26 & 4869.27302 & -3739.81 \\
\hline 27 & 5073.594004 & -3915.07 \\
\hline 28 & 5264.450334 & -4091.34 \\
\hline 29 & 5430.746378 & -4265.67 \\
\hline 30 & 5592.865348 & -4436.68 \\
\hline
\end{tabular}

application on any other field where there is a phenomenon that evolves in a dichotomist way.

The proposal applicative example is on temporary disability insurance. Unfortunately, we did not have real data. But we think that this example can show how it will be possible to apply our model in real life models.

It is also to outline that the alternating compound renewal processes can be applied in many other fields. For example, the application in the reliability of complex mechanical systems is really immediate where the two states are the Up (working) and Down (not working) state. Furthermore, the possibility to put a negative reward for the Down state and a positive reward for the Up state gives the possibility to evaluate costs and revenues by means of a really simple model.

The discrete time approach does not present any applicative difficulty. It is really simple to apply and as proved in D'Amico et al. [11] the strict connection between continuous and discrete time justifies the general application of the discrete time approach.

In future papers, the authors would study the continuous approach of the alternating compound process and the different kinds of evolution equations of these families of
TABLE 7: Fourth case results.

\begin{tabular}{|c|c|c|}
\hline Time & $\begin{array}{c}\text { Premium } 350 € \\
V_{Y}\end{array}$ & $\begin{array}{c}\text { Benefit } 3000 € \\
V_{Z}\end{array}$ \\
\hline 0 & 0 & 0 \\
\hline 1 & 47.33149028 & 0 \\
\hline 2 & 62.84599162 & -5.19786 \\
\hline 3 & 83.46523439 & -20.2702 \\
\hline 4 & 272.2629137 & -37.3761 \\
\hline 5 & 418.2287457 & -69.5687 \\
\hline 6 & 678.0924135 & -145.682 \\
\hline 7 & 807.3802978 & -259.751 \\
\hline 8 & 1161.128492 & -390.073 \\
\hline 9 & 1480.09329 & -536.734 \\
\hline 10 & 1793.079641 & -722.296 \\
\hline 11 & 2157.588588 & -945.753 \\
\hline 12 & 2467.031522 & -1175.66 \\
\hline 13 & 2828.895905 & -1417.62 \\
\hline 14 & 3238.945839 & -1665.04 \\
\hline 15 & 3536.980259 & -1924.8 \\
\hline 16 & 3636.945167 & -2202.5 \\
\hline 17 & 3759.535708 & -2449.91 \\
\hline 18 & 3904.42375 & -2601.93 \\
\hline 19 & 4073.122763 & -2714.58 \\
\hline 20 & 4266.240988 & -2831.77 \\
\hline 21 & 4481.164633 & -2957.59 \\
\hline 22 & 4709.61485 & -3094.52 \\
\hline 23 & 4945.395553 & -3243.85 \\
\hline 24 & 5188.321212 & -3402.87 \\
\hline 25 & 5435.72349 & -3568.57 \\
\hline 26 & 5680.818524 & -3739.81 \\
\hline 27 & 5919.193004 & -3915.07 \\
\hline 28 & 6141.858723 & -4091.34 \\
\hline 29 & 6335.870774 & -4265.67 \\
\hline 30 & 6525.009573 & -4436.68 \\
\hline
\end{tabular}

processes. Moreover, they will try also to generalize these models in a nonhomogenous setting.

\section{Conflict of Interests}

The authors declare that there is no conflict of interests regarding the publication of this paper.

\section{Acknowledgment}

The authors are grateful to the anonymous referee that with his remarks gave the possibility to improve in substantial way the quality of the paper.

\section{References}

[1] P. Lundberg, "On the theory of reinsurance (1909)," in History of Actuarial Science, S. Habermann and T. A. Sibbett, Eds., vol. 7, pp. 291-244, 1995.

[2] H. Cramér, On the Mathematical Theory of Risk, Skandia Jubilee, Stockholm, Sweden, 1930. 
[3] H. Cramér, Collective Risk Theory, Skandia Jubilee Volume, Stockholm, Sweden, 1955.

[4] D. R. Cox, Renewal Theory, Methuen, London, UK, 1962.

[5] F. Gismondi, J. Janssen, and R. Manca, "Non-homogeneous time convolutions, renewal processes and age dependent mean number of motorcar accidents," Annals of Actuarial Sciences, vol. 9, no. 1, pp. 36-57, 2014.

[6] S. Zacks, "Generalized integrated telegraph processes and the distribution of related stopping times," Journal of Applied Probability, vol. 41, no. 2, pp. 497-507, 2004.

[7] A. Di Crescenzo, A. Iuliano, B. Martinucci, and S. Zacks, "Generalized telegraph process with random jumps," Journal of Applied Probability, vol. 50, no. 2, pp. 450-463, 2013.

[8] E. A. Elsayed, Reliability Engineering, John Wiley \& Sons, London, UK, 2nd edition, 2012.

[9] C. M. Ramsay, "The asymptotic ruin problem when the healthy and sick periods form an alternating renewal process," Insurance: Mathematics and Economics, vol. 3, no. 2, pp. 139-143, 1984.

[10] F. Adekambi and S. Mamane, "Health care insurance pricing using alternating renewal processes," Asia-Pacific Journal of Risk and Insurance, vol. 7, no. 1, article 5, 2013.

[11] G. D’Amico, F. Gismondi, J. Janssen, and R. Manca, "Disability insurance claims study by a homogeneous discrete time alternating renewal process," in Modern Problems in Insurance Mathematics, D. Silvestrov, Ed., EAA Series, pp. 187-196, Springer, New York, NY, USA, 2014.

[12] W. Feller, An Introduction to Probability Theory and Its Applications, vol. 1, John Wiley \& Sons, New York, NY, USA, 2nd edition, 1957.

[13] H. C. Tijms, A First Course in Stochastic Models, John Wiley \& Sons, New York, NY, USA, 2003.

[14] F. Beichelt, Stochastic Processes in Science, Engineering and Finance, Chapman \& Hall, New York, NY, USA, 2006.

[15] E. E. Alvarez, "Maximum likelihood estimation in alternating renewal processes under window censoring," Stochastic Models, vol. 22, no. 1, pp. 55-76, 2006.

[16] M. Vlasiou, "Renewal processes with costs and rewards," 2010, http://www.win.tue.nl/ mvlasiou/documents/publications/renewals.pdf.

[17] G. Corradi, J. Janssen, and R. Manca, "Numerical treatment of homogeneous semi-Markov processes in transient case-a straightforward approach," Methodology and Computing in Applied Probability, vol. 6, no. 2, pp. 233-246, 2004.

[18] J. Janssen and R. Manca, Applied Semi-Markov Processes, Springer, New York, NY, USA, 2006. 


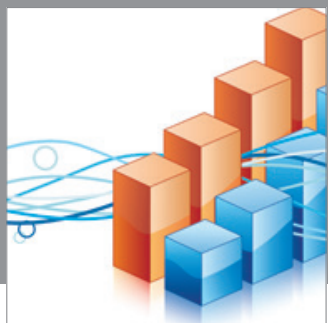

Advances in

Operations Research

mansans

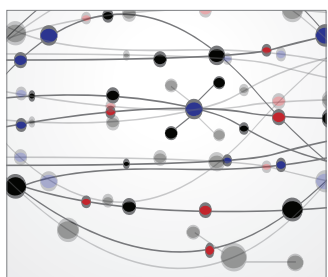

The Scientific World Journal
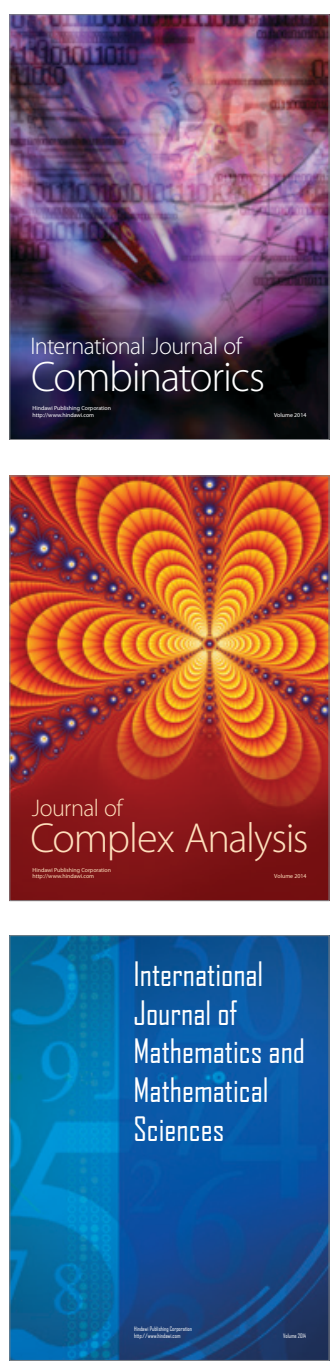
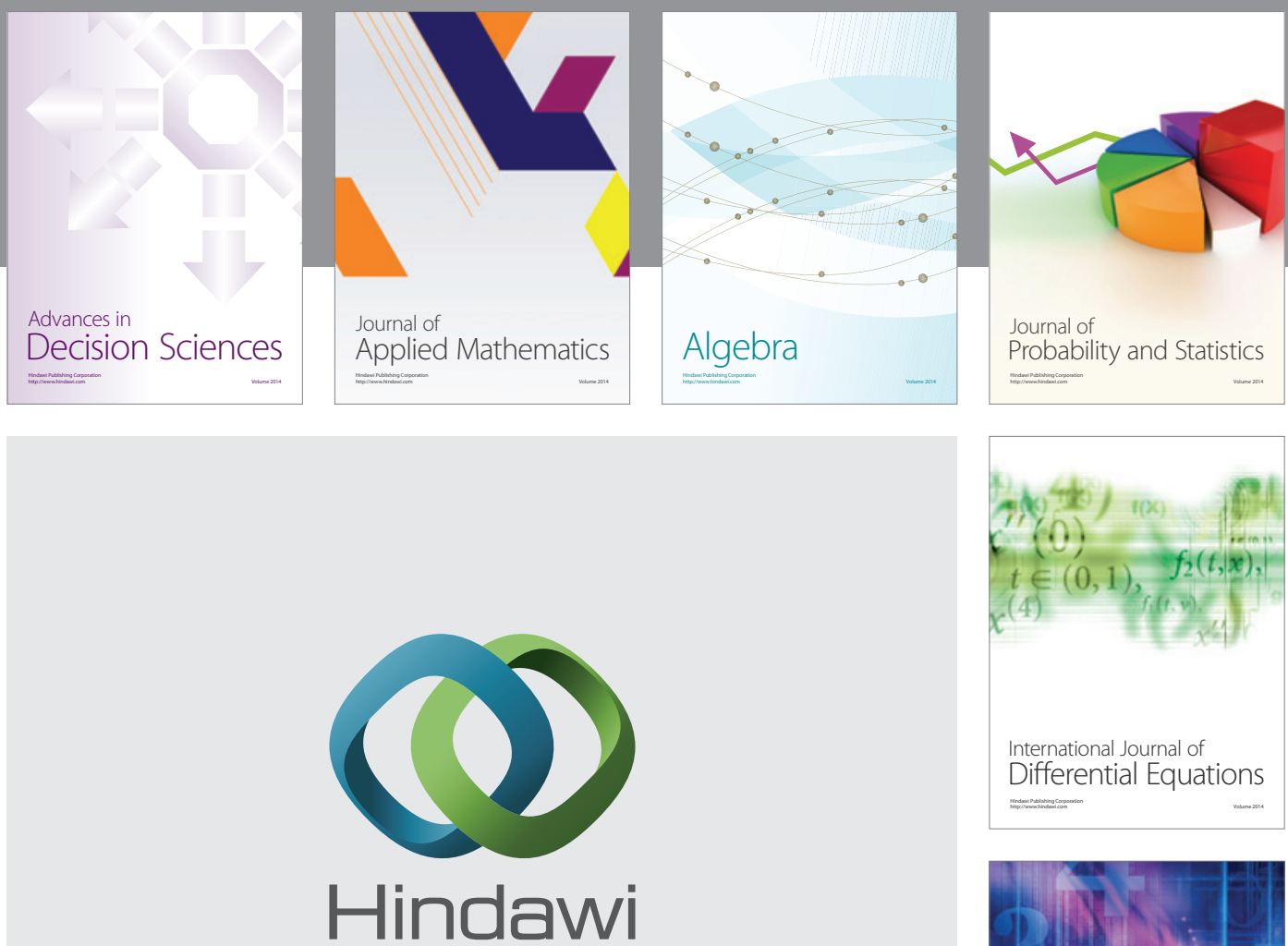

Submit your manuscripts at http://www.hindawi.com
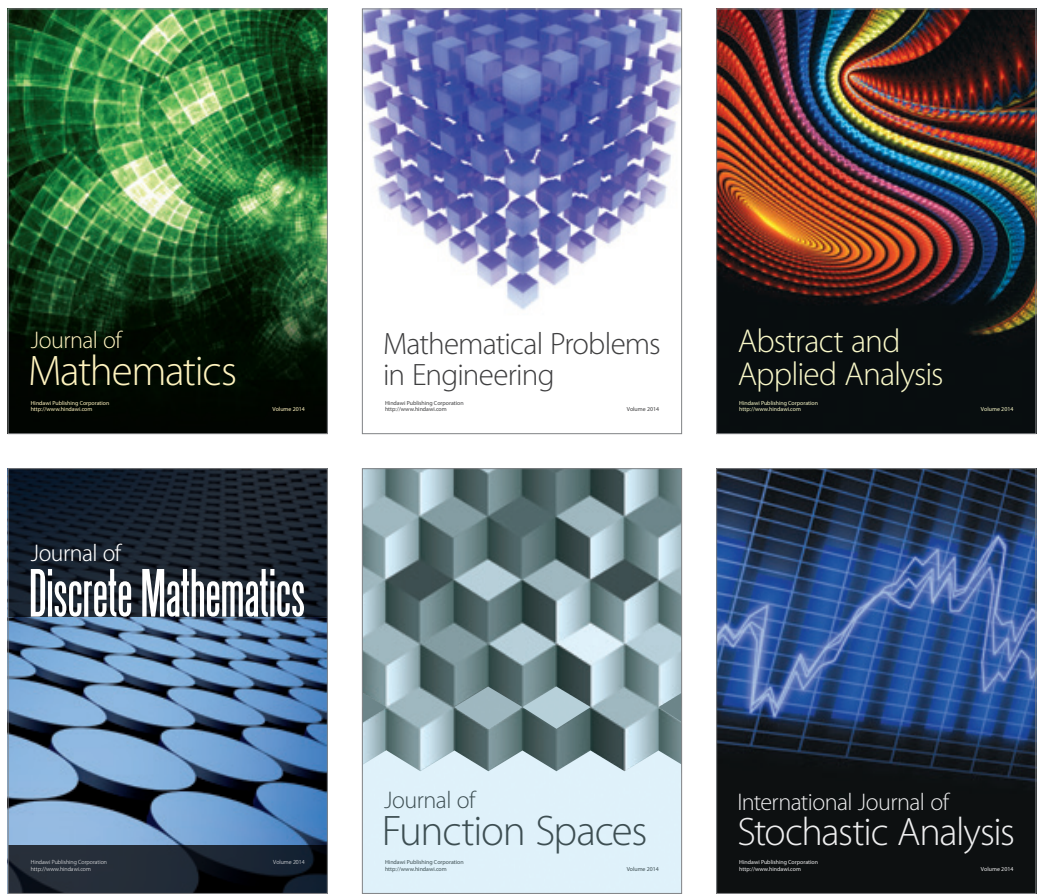

Journal of

Function Spaces

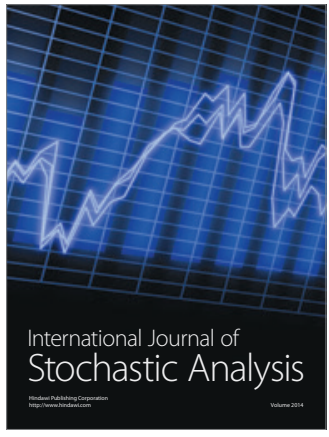

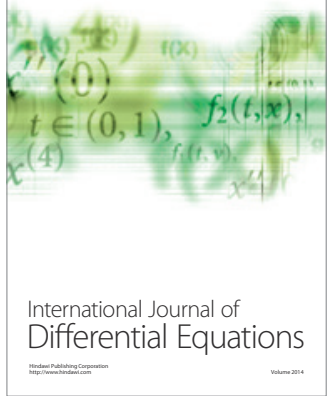
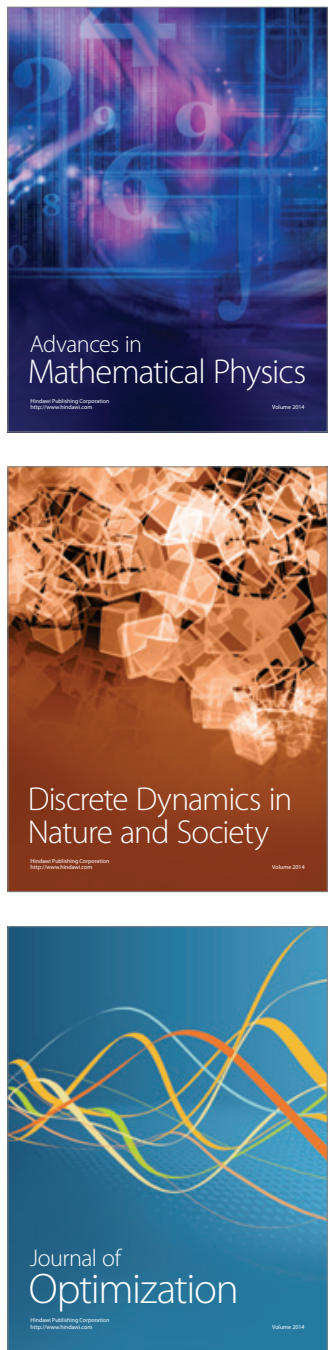